\title{
Psychosocial intervention in at-risk adolescents: using event-related potentials to assess changes in decision making and feedback processing
}

\author{
H.L. Pincham¹, D. Bryce ${ }^{2}$ P. Fonagy ${ }^{1,3}$ \& R.M.P Fearon ${ }^{1,3}$
}

${ }^{1}$ Developmental Neuroscience Unit, Anna Freud National Centre for Children and Families

${ }^{2}$ Department of Psychology, University of Tübingen

${ }^{3}$ Research Department of Clinical, Educational and Health Psychology, University College London

\section{Corresponding author details:}

Pasco Fearon

Email: p.fearon@ucl.ac.uk

Phone: +442076791244

Key words: adolescent, event-related potential, intervention, psychosocial, FRN, LPP

Acknowledgments: We would like to thank all of the participants, schools and key workers who took part in this study. We would like to especially thank the following individuals for their assistance with data collection and/or preparing the data for analysis: Charlotte Bargus, Elizabeth Harding, Danae Kokorikou, Jodie Walman and Anna Zonderman. This work was supported by the Waterloo Foundation (grant code 564/709) and Kids Company, London.

\begin{abstract}
Decision making and feedback processing are two important cognitive processes that are impacted by social context, particularly during adolescence. The current study examined whether a psychosocial intervention could improve psychological wellbeing in at-risk adolescent boys, thereby improving their decision making and feedback processing skills. Two groups of at-risk adolescents were compared: those who were relatively new to a psychosocial intervention, and those who had engaged over a longer time period. Electroencephalography was recorded while the young people participated in a modified version of the Taylor Aggression Paradigm. The late positive potential (LPP) was measured during the decision phase of the task (where participants selected punishments for their opponents). The feedback related negativity (FRN) and P3 components were measured during the task's outcome phase (where participants received 'win' or 'lose' feedback). Adolescents who were new to the intervention (the minimal-intervention group) were harsher in their punishment selections than those who had been engaged in the program for much longer. The minimalintervention group also showed an enhanced LPP during the decision phase of the task, which may be indicative of immature decision making in that group. Analysis of the FRN and P3 amplitudes revealed that the minimal-intervention group was physiologically hypo-sensitive to feedback, compared with the extended-intervention group. Overall, these findings suggest that long-term community-based psychosocial intervention programs are beneficial for at-risk adolescents, and that event-related potentials can be employed as biomarkers of therapeutic change. However, because participants were not randomly allocated to treatment groups, alternative explanations cannot be excluded until further randomized controlled trials are undertaken.
\end{abstract}


Adolescence is a time of rapid cognitive and behavioural maturation. However, some cognitive skills - such as decision making and feedback processing - are sub-optimal during adolescence. This may be partly due to the fact that adolescents are highly susceptible to influence from their social environment, which affects the way that they make decisions and how they respond to the outcomes of their decisions and behaviour. Arguably, the impact of social context is crucially important for 'at-risk' adolescents, whose decisions often lead to poor psychological and behavioural outcomes $[1,2]$. The current study aimed to assess decision making and feedback processing in at-risk adolescent boys, by employing a sensitive physiological measure. To that end, electroencephalography was recorded while two groups of young people participated in a version of the Taylor Aggression Paradigm (TAP). The two groups differed in their degree of involvement in a psychosocial intervention program, thereby allowing us to explore the relationship between therapeutic intervention, decision making and feedback processing in at-risk young people.

We recently demonstrated the importance of social context on decision making and feedback (outcome) processing in typically-developing adolescents $[3,4]$. Using a modified TAP task, participants competed online against two peer opponents. Unbeknownst to participants, the opponents were fictional - we created one highly provocative opponent and one less provocative opponent. On each trial of the task, participants either won or lost to their opponent (enabling us to examine feedback processing). In addition, participants were able to select the level of punishment experienced by their opponent (allowing us to examine decision making). Electrical brain activity was recorded using electroencephalography, and event-related potentials (ERPs) were compared across older and younger age groups (1012 years, versus 14-16 years). During the Decision Phase of the task (when participants decided how harshly to punish their opponent), we examined the Late Positive Potential (LPP) component, as an electrophysiological index of participants' emotional processing whilst making their decision. The LPP was larger when adolescents competed against the highly provocative opponent versus the less provocative opponent. This result was restricted to younger adolescents suggesting that emotional reactivity during the Decision Phase was heightened for younger, more immature adolescents. During the Feedback Phase of the task, the Feedback Related Negativity (FRN) component suggested that adolescents place a greater value on winning when competing against a highly provocative opponent than when competing against a less provocative opponent. This was because the FRN was reduced (less negative) for high provocation trials versus low provocation trials. Collectively, the data highlight the relevance of social context when adolescents make decisions and receive feedback. The results also demonstrate how ERPs can be successfully used to examine the trajectory of normal adolescent development. The current study therefore asked whether the application of these biomarkers could be further extended to assess changes in cognitive processing associated with therapeutic interventions - rather than to simply assess normative adolescent development. Specifically, we recruited at-risk adolescents, whose decision making skills and feedback processing are likely to be heavily influenced by social context. 
At-risk adolescents do not represent a homogenous psychiatric population but often live in high stress environments characterised by poverty, community violence and trauma [5]. These young people present with a heterogeneous range of cognitive and behavioural difficulties, particularly conduct problems and antisocial, externalizing behaviors [5,6]. ERPs are useful measures to assess functioning in children and young people who have experienced early adversity and psychosocial deprivation [7,8]. Our own work with at-risk adolescents has confirmed the efficacy of ERPs to assess psychopathology and therapeutic change in that population. For example, using a passive picture viewing task, we demonstrated that the LPP was reduced (less positive) in adolescent boys who had recently engaged in criminal activities compared with adolescents who had not [9]. Using the same passive viewing task, we showed that the LPP was reduced in adolescent boys who were new to a community psychosocial intervention, versus those who had received the intervention for approximately one year [6]. In other words, the LPP may have been altered by therapeutic intervention. In the current study, we aimed to extend our work investigating the value of the LPP as a biomarker of therapeutic change by measuring this component while participants actively made decisions (using the TAP task), rather than while viewing emotional images passively. We also aimed to investigate how informative the FRN is as a potential biomarker of therapeutic intervention related to changes in reward processing, by measuring this component in the TAP task. Two groups of at-risk adolescents were compared: those who were relatively new to a longterm psychosocial program, versus those who had engaged for a longer period. It is important to highlight that this cross-sectional design cannot assess within-subject variability, and cannot speak directly to intervention-related change. However, participants in the two groups were well matched on numerous social and demographic variables, such that reliable differences between the groups should support the use of ERP biomarkers as indices of therapeutic effectiveness - which could then be further tested in randomized controlled trials (RCTs).

A number of clinical interventions for at-risk adolescents have been reported, but their outcomes are mixed [10-15]. The mechanisms underpinning successful treatments are often unclear, and so it is argued that research should use sensitive methodological techniques to help reveal underlying mechanisms $[16,6,17,18]$. The current study therefore employed ERPs to examine whether decision making and feedback processing differ as a result of involvement in psychosocial intervention. Specifically, we recruited two groups of at-risk adolescent boys: those who were new to a psychosocial program and had received minimal therapeutic input, and those who had been engaging with a therapeutic keyworker as part of the intervention for over one year, on average. Clearly, a longitudinal RCT would be a superior research design to examine our research question. Yet since RCTs are highly expensive and resource intensive, we argue that observing reliable differences between these two groups is an important initial step to establish 'proof of principle' that psychosocial interventions have beneficial effects for high risk teenage boys. The current study would therefore be beneficial prior to undertaking fully randomized trials. 
The community-based intervention assessed in this study supports young people with complex social needs including poverty and disruptive behavior. Cognitive and behavioural impairments in these adolescents are not unexpected, given that chronic social adversity is correlated with conduct problems and psychological ill-health [19,20]. Decision making and feedback processing therefore seem to be appropriate targets for assessing the biological changes associated with therapeutic intervention in at-risk adolescents. Existing research supports this line of reasoning because adolescents with disruptive behaviour disorders and externalizing symptoms show abnormal feedback processing: they typically choose large immediate rewards even if their choices are associated with large potential punishments [21]. These young people will also perseverate in responding to rewarding feedback, even when doing so becomes unfavourable because response contingencies change [22,23]. Using functional magnetic resonance imaging, feedback processing has been shown to be deficient in young people with antisocial behavior, as evidenced by abnormal activity in the ventral striatum [24], the caudate [25,26], the anterior cingulate [24,27] and the orbito-frontal cortex [28,29]. In EEG studies, the FRN component has been found to be altered in adolescent participants with externalising behaviours. For example, adolescents with foetal drug exposure show high risk-taking behaviours and reduced (less negative) FRN amplitudes [30]. In a different study, FRN amplitudes evoked by failure were reduced in 15 year old boys with high levels of sensation seeking [31]. This result is consistent with the theory that high risk adolescents are physiologically hypo-sensitive to feedback, but is in contrast to data from typically-developing adolescents, who are often hypersensitive to feedback information (particularly rewards) [32]. Research by Krämer, Büttner, Roth and Münte [33] used the TAP task and found that the difference between reward-related FRN amplitudes on win versus loss outcomes was larger in aggressive adults versus non-aggressive adults. Taken together, the existing research confirms that feedback processing is disrupted in participants with externalizing behaviours. However, it is currently unclear whether psychosocial intervention impacts FRN magnitude in at-risk individuals, and therefore whether the FRN can be employed as a biomarker of therapeutic change.

Given that adolescents with externalizing behaviours may show attentional hypo-sensitivity to feedback information, we also measured the feedback-related P3 component in this study. Reduced (hypo-sensitive) P3 amplitudes have been linked to various psychopathologies including antisocial behavior as well as depression, schizophrenia, and dependency disorders [34-38]. The P3 is frequently examined in studies of feedback processing because it is sensitive to both feedback outcome (for example, win versus loss outcomes) and feedback magnitude (for example, amount of money won) [39-43].

To achieve this study's aims, we invited at-risk adolescents who were involved in a long-term therapeutic psychosocial intervention (provided by a charity based in inner city London) to participate. The intervention aimed to help at-risk children and adolescents suffering from chronic social adversity. As part of the intervention, at-risk young people were paired with an adult keyworker, who was trained in principles of attachment theory and behavior 
management. The keyworker coordinated a long-term, broad package of care for the young person, including practical help and emotional support. The package of care was designed to help adolescent development over an extended period of time (months to years), by enhancing psychological well-being, promoting healthy attachments, reducing externalizing behaviour, increasing educational attainment, improving physical health and helping to mediate povertyrelated socio-legal problems (such as access to housing) [44,45].

The two groups of at-risk adolescents participated in the TAP task while their brain activity was recorded using EEG. It is important to reiterate that the recruited sample did not reflect a homogenous psychiatric population. However, we claim that it is vital to conduct research with at-risk adolescents who are lacking a medical diagnosis, as these young people are often excluded from preventative services and psychological therapeutic provisions. We hypothesized that psychosocial intervention would normalize feedback processing and would be associated with a more mature decision making style. Therefore, it was predicted that the minimal-intervention participants would select harsher punishments for their opponents on the TAP task than would extended-intervention participants. During the Decision Phase, we predicted that the LPP would be larger (more positive) for minimal-intervention participants compared with extendedintervention participants (consistent with our prior research using a normative adolescent sample, which demonstrated a larger LPP during the Decision Phase for younger, more immature participants). During the Feedback Phase, we hypothesized that the FRN and P3 components would be reduced for the minimal-intervention group versus the extended-intervention group, consistent with prior research demonstrating physiological hypo-sensitivity to feedback in externalizing participants [see 30,31].

\section{Materials \& Methods}

\section{Participants}

Participants were 77 adolescent males (aged 13-18 years) who had been assessed by the charity providing the intervention as having 'High' or 'Medium' levels of risk. High or Medium risk ratings required at least one of the following criteria to be met: emotional turmoil, serious risk-taking behaviour, homelessness, criminal behaviour, signs of neglect and/or abuse. Of these 77 participants, one did not complete the task, the equipment malfunctioned during one further session and three more were excluded due to poor EEG data quality. The remaining participants were matched for age across the minimal-intervention and extended-intervention groups, resulting in the exclusion of one additional participant who could not be age-matched across the groups. This resulted in 36 participants per group.

Young people were eligible for participation in the study if they had no developmental disability or delay (for example, learning difficulties or autism spectrum disorder). In addition, participants were required to be academically eligible for mainstream state schooling in England. Although a high proportion of participants had been excluded from 
school at some point in their education, this was not due to intellectual abilities, but typically for antisocial behaviour or failing to follow school rules.

The intervention was a long-term psycho-social package of care coordinated by a designated keyworker at 'Kids Company, London'. Participants in the minimal-intervention group had been involved in the psychosocial intervention for four months or less $(\mathrm{Mean}=1.9, \mathrm{SD}=1.7$, range=0-4.6), and participants in the extended-intervention group had been involved in the intervention for nine months or more (Mean=16.6, $\mathrm{SD}=8.4$, range=9.4-36.5). Keyworkers confirmed that all extended-intervention participants had regularly engaged in the intervention program.

Socio-economic status was estimated using a freely available tool from the Office for National Statistics (UK). The tool uses postcode data to estimate the proportion of households living in poverty (that is, below $60 \%$ median income) for small geographic areas. Estimates are derived using survey, census and administrative data. As shown in Table 1, socio-economic status did not differ across the two groups.

Given the cross-sectional design of the study, it was important to document the nature of participants' emotional and behavioral difficulties before they received psychosocial intervention. Keyworkers were therefore interviewed to estimate the participants' initial emotional and behavioral difficulties. Keyworkers indicated whether each participant had been involved in criminal behavior, had been excluded from school due to antisocial behavior and/or had experienced trauma (for example: physical, emotional, sexual abuse; maltreatment, neglect; bereavement of a close caregiver; exposed to violence; raised in foster care). Ratings were retrospective, as keyworkers documented the nature of the young persons' difficulties prior to intervention. Because the minimal-intervention participants were new to the therapeutic program, 11 of those participants had not yet received a keyworker at the time of participation. Case file records were therefore assessed to establish those participants' emotional and behavioral difficulties, and missing scores were recorded for inconclusive case files.

All participants provided written, informed consent and parental/guardian consent was additionally obtained for participants under 16 years of age. Participants had normal or corrected-to-normal vision, and none disclosed a developmental disability. Participation was compensated with a $£ 30$ shopping voucher for the entire study session (which included this task, plus another task previously reported [6]). This sum of remuneration was determined in consultation with key workers and pilot participants.

\section{Stimuli \& Procedure}

All stimuli were presented on a black background on a Dell 17-inch monitor, refreshing at $60 \mathrm{~Hz}$. In the Decision Phase, the stimulus of interest was the word 'Think', which appeared in the centre of the screen in white text (visual angle: $4^{\circ} \times 1^{\circ}$, at a viewing distance of $57 \mathrm{~cm}$ ). In the Outcome Phase, the stimuli of interest were images of a green tick inside a white box (indicating a win outcome) or a red cross inside a white box (indicating a loss outcome). 
The Outcome Phase stimuli appeared in the centre of the screen and assumed a visual angle of $6.5^{\circ} \times 6.5^{\circ}(57 \mathrm{~cm}$ viewing distance). On trials where the participant experienced a loss outcome, an aversive auditory stimulus (white noise) was delivered via headphones after the offset of the visual cross stimulus. The white noise increased in volume with increasing punishment level. The experiment was programmed and presented using E-Prime 2.0 software (Psychology Software Tools, Pittsburgh, PA).

A modified Taylor Aggression Paradigm [4] was used to examine decision making and feedback processing. Participants were told that they would compete against two other players in an online, multi-player game. The competitive game that participants played against their opponents was a simple Go/No-go task. Participants were informed that their performance on the Go/No-go game would determine whether they won or lost a given trial. However, the social context was fictional: the computer program provided pre-determined responses on behalf of each opponent, and the feedback that participants received on each round (win or loss) was fixed, and not determined by participants' responses. Efforts were taken to ensure the believability of the social context, including the experimenters calling a fictional lab to arrange the online connection, and participants being introduced to their opponents via a fictional web-camera. Participants were shown two opponents (pre-recorded actors) who also wore an EEG net and waved at them before the onset of the first block. Both opponents were males, who appeared to be approximately the same age as the participants. To enhance believability, a break block was included, and participants were told that during this time their two opponents were playing against each other. Participants appeared to believe the cover story, and were very engaged in the task.

On each trial, participants experienced the following sequence of events, as shown in Figure 1: an instruction to think about the punishment level they would like to select for their opponent on this trial (Decision Phase), followed by actual punishment selection by participants for their opponent, followed by the competitive Go/No-go game against their opponent, followed by win or loss feedback (Outcome Phase), followed by a display of the level of punishment selected by the opponent for the participant, followed by white noise (if the participant lost that trial). During the Decision Phase, the 'Think' stimulus appeared on the screen for $1500 \mathrm{msec}$. The participant then selected a potential punishment for their opponent (from levels 1 to 6, via a keyboard button press) and was led to believe that the selected punishment would be administered to their opponent if the opponent lost the trial. During the Outcome Phase, the tick or cross stimulus appeared in the center of the screen for $1000 \mathrm{msec}$ to indicate whether the participant had won or lost that trial. Participants were told that performance on the trial (win or loss) was determined by their speed and accuracy. Following the outcome stimulus, the participant was then informed about the level of punishment that their opponent had selected to inflict on them at the beginning of that trial.

On each trial, participants experienced either a win or loss outcome. The task was designed so the distribution of win/loss outcomes was $50 \%$ win and $50 \%$ loss for every block. Participants were told they started with $£ 5$ 'in the 
bank'. Win outcomes were rewarded with $35 \mathrm{p}$. Loss outcomes were punished with a monetary loss and an aversive auditory stimulus, both of which increased (in value / amplitude) with the punishment level. Punishment levels 1 to 6 were respectively associated with punishments of $10 \mathrm{p}, 20 \mathrm{p}, 30 \mathrm{p}, 40 \mathrm{p}, 50 \mathrm{p}$ or $60 \mathrm{p}$. Participants believed that the magnitude of the punishment they experienced was selected by their opponent during the Decision Phase. In reality, the punishments experienced by participants were predetermined by the experimental software: one opponent was a 'low provoker' (mean punishment $=23 \mathrm{p}$ ) and the other was a 'high provoker' (mean punishment=42p). All participants initially competed against the low provoker. The type of opponent (low provoker vs. high provoker) alternated across 4 blocks of 20 trials per block.

INSERT FIG 1 HERE. Fig 1 Temporal outline of an experimental trial. Participants were asked to think about punishment for their opponent, prior to selecting a punishment (between 10p - 60p) for their opponent. Participants then played a Go/No-go game against their opponent. A win or loss outcome stimulus was then displayed, and finally the opponent's punishment selection was revealed. The participant was credited with $35 \mathrm{p}$ on every 'Win' trial, and was debited 10p-60p on every 'Loss' trial. ERPs were locked to the onset of the Decision Phase or the Outcome Phase, and these are highlighted in gray.

\section{Questionnaire Measures}

Early Adolescent Temperament Questionnaire: participants completed a short form of the revised Early Adolescent Questionnaire [46]. Sub-scale scores were calculated for Attention, Aggression, Frustration and Inhibitory Control.

Strengths and Difficulties Questionnaire: participants provided self-report responses to the Strengths and Difficulties Questionnaire [47]. This measure is appropriate for community samples. Sub-scale scores were calculated for pro-social behaviour, hyperactivity, emotional symptoms, conduct problems and peer problems.

Inventory of Callous and Unemotional Traits: participants provided self-report responses to the Inventory of Callous and Unemotional Traits. Measures on three sub-scores were calculated: callousness, unemotional, uncaring. This measure has been validated with community samples of adolescents [48].

\section{EEG Acquisition and Pre-processing}

The 129-channel Hydrocel geodesic sensor system (Electrical Geodesics Inc.) was used to record EEG at a sampling rate of $250 \mathrm{~Hz}$. An anti-aliasing low-pass filter $(70 \mathrm{~Hz})$ was applied during data acquisition. Data were bandpass filtered between 0.1 and $30 \mathrm{~Hz}$ offline and recomputed to an average reference. Interpolation was applied to individual channels using the spline method. For the minimal-intervention group, the mean percentage and range of interpolated channels was $8.25 \%$ (range: $0.78-10.08 \%$ ). For the extended-intervention group, the mean percentage and 
range of interpolated channels was 5.32\% (range: 0-9.30\%). Independent component analysis removed stereotyped artifacts using the FASTER toolbox [49]. Epochs were created between -200 and $700 \mathrm{~ms}$ relative to the epoching stimulus. Epochs were rejected as artifacts if they met either of the following criteria: voltage deviations exceeding $150 \mu \mathrm{V}$ relative to baseline or peak-to-peak moving amplitude exceeding $150 \mu \mathrm{V}$ in a $200 \mathrm{msec}$ moving window.

\section{Data Analysis}

We examined whether the two groups were initially comparable using independent samples t-tests to compare age and socio-economic status. Chi-square tests compared the proportion of participants who had experienced school exclusion, criminal engagement and trauma.

\section{Behavioural Data}

The punishment level selected by participants on the first trial of the experiment was used as a proxy for unprovoked aggression, and compared across the two groups using an independent-samples t-test. First punishment responses have been successfully used to reflect unprovoked aggression in previous research [50]. Each participant's average punishment selections were then computed for every block of the task. Punishment selections were analysed using an ANOVA with provocation (low vs. high) as a within-subjects factor and group (minimal-intervention vs. extended-intervention) as a between-subjects factor. Finally, we calculated how quickly participants made their punishment selections, and subjected these reaction time data to an ANOVA with provocation and group as factors.

To investigate group differences in inhibitory control, we analysed data from the Go/No-go game. Specifically, the proportion of correct 'Go' responses, the proportion of correct 'No-go' responses, and reaction times to 'Go' stimuli were calculated and compared across the two groups using independent-samples t-tests.

\section{Questionnaire Measures}

Group scores on each sub-scale of the Early Adolescent Temperament Questionnaire, the Strengths and Difficulties Questionnaire and the Inventory of Callous and Unemotional Traits were contrasted using independent-samples t-tests.

\section{ERP Data: Decision Phase}

ERP data from the Decision Phase and the Outcome Phase of the task were analysed separately. ERPs were locked to the onset of the Think screen in the Decision Phase and the onset of the Outcome screen in the Outcome Phase.

In the Decision Phase, we used the LPP as a determinant of punishment selection [3]. The LPP was defined as activity in a parietal scalp location: electrodes 53, 54, 60, 61, 67 (left hemisphere) and 77, 78, 79, 85, 86 (right 
hemisphere) in the hydrocel geodesic sensor net. As per Cuthbert et al. [51], the LPP was defined as mean amplitude in the window 400-700msec after onset of the stimulus (the Think screen). Mean LPP amplitudes were analysed using an ANOVA with hemisphere, provocation and group as factors.

\section{ERP Data: Outcome Phase}

In the Outcome Phase, ERPs were locked to the onset of the Win or Loss outcome screens. In line with prior findings $[52,53]$, activity at FCz (electrode 6 in the hydrocel geodesic sensor net) in the time window 200-300msec after stimulus onset was used to calculate the FRN. FRN mean amplitudes were analysed using an ANOVA with outcome (win vs. loss), provocation (low vs. high) and group (minimal-intervention vs. extended-intervention) as factors.

In line with previous work, the P3 was defined as the positive deflection occurring in a central-parietal scalp area (electrodes 61, 62, 67, 72, 77, 78 in the hydrocel geodesic sensor net) [54-56]. Mean P3 amplitudes were calculated in the window 300-500msec after stimulus onset, as per previous research [57-59]. P3 mean amplitudes were subjected to an ANOVA with outcome, provocation and group as factors.

\section{Results}

\section{Sample Characteristics}

The minimal-intervention and extended-intervention groups were matched for age and socio-economic status. According to keyworker ratings, the groups were initially equated in terms of the percentage of participants who had been excluded from school, engaged in criminal activity, and experienced trauma. Self-report questionnaire measures indicated that psychological functioning was similar between the two groups at the time of participation in the study, as there were no significant group differences on any questionnaire sub-scale (see Table 1). Demographic details, behavioural characteristics and questionnaire data are presented in Table 1.

INSERT TABLE 1 HERE. Table 1 Demographic and behavioural characteristics (mean and standard deviations, or percentages) for the minimal-intervention and extended-intervention groups ( $\mathrm{N}=36$ per group).

\section{Behavioural Data}

The first punishment selected by each participant was used as an index of unprovoked aggression. As shown in Table 2, first punishment selections did not differ between the two groups $(t(70)=1.350, p=.181)$. For both groups, average punishment selections were larger under high provocation compared with low provocation $(F(1,70)=74.270$, $\left.p<.001, \eta^{2}=.515\right)$. Importantly, an interaction between group and provocation emerged $(F(1,70)=5.019, p=.028$, 
$\left.\eta^{2}=.067\right)$. As shown in Figure 2, punishment selections did not differ between minimal-intervention and extendedintervention participants under low provocation. Under high provocation, however, minimal-intervention participants selected harsher punishments. Overall, participants were faster to select punishments when competing against the highly provocative player $\left(F(1,70)=5.209, p=.026, \eta^{2}=.069\right)$, but reaction times did not vary as a function of group $(F<1)$.

INSERT FIG 2 HERE. Fig 2 Mean punishment selections for the minimal-intervention and extended-intervention groups under high and low provocation.

Behavioural data from the Go/No-go game was used to assess underlying group differences in inhibitory control. Accuracy on Go trials did not differ between the two groups (Minimal-intervention: 89.32\% (SE: 1.66); Extended-intervention: $86.03 \%$ (SE: 2.31$) ; t(70)=1.158, p=.251$ ). Accuracy on No-go trials was also statistically equivalent across the two groups of participants (Minimal-intervention: 99.17\% (SE: 0.21); Extended-intervention: $98.89 \%$ (SE: 0.51); $t(70)=.506, p=.614)$. Finally, reaction times to the Go stimuli did not differ as a function of intervention (Minimal-intervention: $474.75 \mathrm{msec}$ (SE: 5.54); Extended-intervention: $478.82 \mathrm{msec}(\mathrm{SE}: 7.82)$; $t(70)=-$ $.425, p=.672)$.

INSERT TABLE 2 HERE. Table 2 Mean behavioural data from the TAP for the minimal-intervention and extendedintervention groups. Participants' punishment selections could range from $10 \mathrm{p}$ to $60 \mathrm{p}$. Higher values are indicative of larger punishment selections for their opponent. Higher reaction times are indicative of slower responses. Standard deviations are shown in brackets.

\section{ERP Data: Decision Phase}

Data from the LPP analyses are shown in in Figure 3. There were no main effects of hemisphere $(\mathrm{F}<1)$, group $\left(F(1,70)=2.346, p=.130, \eta^{2}=.032\right)$ or provocation $\left(F(1,70)=3.006, p=.087, \eta^{2}=.041\right)$. However, the provocation $\times$ group interaction approached significance $\left(F(1,70)=3.776, p=.056, \eta^{2}=.051\right)$, and the three-way interaction between hemisphere, provocation and group was statistically significant $\left(F(1,70)=5.554, p=.021, \eta^{2}=.074\right)$. Post-hoc contrasts were Bonferroni corrected for multiple comparisons (critical $\alpha=.025$ ). Although the LPP did not vary with provocation for the extended-intervention group ( $\mathrm{p}=.883$ ), the LPP was larger (more positive) under high provocation versus low provocation for the minimal-intervention group $(\mathrm{p}=.011)$. This effect was stronger in the left hemisphere $(\mathrm{p}=.004)$ than the right hemisphere $(\mathrm{p}=.072)$.

INSERT FIG 3 HERE. Fig 3 The LPP as a function of group and provocation condition, time-locked to the onset of the 'Think' screen. ERPs are shown at parietal electrodes (black circles on the topographies indicate the electrodes 
used). Grey boxes show the time window (400-700msec) used to calculate mean amplitudes. Topographies show the scalp distribution of the difference in amplitude between provocation conditions (low provocation minus high provocation).

\section{ERP Data: Outcome Phase}

As hypothesized, the FRN was more negative following loss outcomes than win outcomes $(F(1,70)=43.308$, $\left.p<.001, \eta^{2}=.382\right)$. Mean FRN amplitudes were also more negative for the extended-intervention group than the minimalintervention group $\left(F(1,70)=4.217, p=.044, \eta^{2}=.057\right.$; see Figure 4). Provocation did not impact FRN amplitude, as no main or interaction effects involving provocation were statistically significant (smallest $p=.272$ ). Further, the group (minimal-intervention versus extended-intervention) $\mathrm{x}$ outcome (win versus loss) interaction was not significant $\left(F(1,70)=1.585, p=.212, \eta^{2}=.022\right)$.

P3 amplitudes were larger (more positive) on win trials compared with loss trials $(F(1,70)=46.409, p<.001$, $\left.\eta^{2}=.399\right)$. P3 amplitudes were also larger for the extended-intervention group compared with the minimal-intervention group $\left(F(1,70)=7.727, p=.007, \eta^{2}=.099\right)$. As shown in Figure 4, a significant interaction between outcome and group suggested that the magnitude of difference between win and loss trials was reduced for the minimal-intervention group $\left(F(1,70)=6.644, p=.012, \eta^{2}=.087\right)$. In other words, minimal-intervention participants appeared hypo-sensitive to feedback stimuli. No main or significant effects involving provocation were statistically significant (smallest $p=.152)$.

INSERT FIGURE 4 HERE. Fig 4 The FRN and P3 ERPs as a function of group and outcome, time-locked to the onset of the feedback stimulus (win or loss). Grey boxes show the time windows used to calculate mean amplitudes (200-300msec for the FRN and 300-500msec for the P3). Topographies show the scalp distribution of the difference in amplitude between outcomes (loss minus win). Black circles on the topographies indicate the electrodes used to generate the ERPs.

\section{Discussion}

The major aim of this study was to investigate whether decision making and feedback processing are impacted by participation in a psychosocial intervention. Assuming that psychosocial intervention improves cognitive and behavioral functioning, we expected to see differences between the minimal-intervention and extended-intervention groups in terms of their behavior (punishment selection choices), as well as their brain responses during the Decision and Outcome Phases of the task. Overall, our data suggest that psychosocial intervention may be associated with improvements in decision making and feedback processing (as reflected by the behavioural and EEG data). Specifically, minimal-intervention participants punished the highly provocative opponent more harshly than extended-intervention 
participants did. In addition, during the Decision Phase, the LPP was effected by provocation for the minimalintervention group only (consistent with immature decision making). The minimal-intervention group also demonstrated reduced FRN and P3 components during the Outcome Phase (consistent with physiological hypo-reactivity to feedback). These findings are of course consistent with a treatment effect, but because the participants were not randomly allocated to the two treatment groups, alternative, non-causal explanations cannot be ruled out until full clinical trials are undertaken. The findings are discussed in turn below.

\section{Behavioural Responses}

For both groups of participants, provocation condition (as administered by the two 'opponents') influenced participants' punishment selections. Participants selected larger average punishments when competing against the highly provocative opponent versus the less provocative opponent [consistent with 33]. Although both groups were impacted by provocation, punishment selection responses were substantially reduced (less harsh) under high provocation for the extended-intervention group. In other words, the extended-intervention group showed reduced retaliatory behavior when placed in a provocative social context. This result suggests that the psychosocial intervention was effective in reducing inter-personal aggressive behavior, and is consistent with other reports of decreased externalizing symptoms following psychosocial intervention [for a review see 60]. Interestingly, harsher punishment selections by minimal-intervention participants appeared to index a reactive response (and not a proactive or unprovoked response) to the social environment because initial punishment selections not differ between the two groups. These punishment selection responses were arguably not due to underlying differences in inhibitory control, as no differences emerged between the two groups on the Go/No-go task.

A complementary explanation for larger punishment selections in the minimal-intervention group is that minimal-intervention participants relied on a more immature decision making strategy. Previous work from our lab demonstrated that younger adolescents (10-12 years) selected harsher average punishments than older adolescents (1416 years), even though initial punishment selections did not differ between the two age groups [3]. In the current study, the behavior of at-risk adolescents who were new to the intervention mirrors that of early adolescents in our previous study, despite the minimal-intervention and extended-intervention groups being tightly matched for age here.

\section{Decision Phase}

In addition to measuring changes in behavior, we measured the LPP during the Decision Phase of the task. Confirming our hypothesis, the minimal-intervention group showed an enhanced (more positive) LPP under high provocation versus low provocation. By contrast, provocation did not affect LPP amplitudes for extended-intervention participants. The LPP is typically larger (more positive) for arousing stimuli, and is argued to index sustained processing 
of arousing information $[61,62,51,63]$. In this manner, our results suggest that the socially provocative nature of the task was more emotionally arousing for minimal-intervention participants. This heightened emotional arousal may explain minimal-intervention participants' harsher punishment choices. These findings resonate with our previous work showing that LPP amplitudes are altered by provocation in younger, but not older, adolescents [3]. Hence, the LPP data reported here suggest immature decision making abilities in the minimal-intervention participants.

\section{Outcome Phase}

During the Outcome Phase of the task, the FRN and P3 components were analyzed to help reveal whether participation in a psychosocial intervention improves young people's responses to feedback information. Our data were consistent with the notion that the FRN indexes emotional appraisal of outcome stimuli, as the FRN is typically larger (more negative) following bad outcomes [64-66]. For both groups of participants, we observed an enhanced FRN following loss outcomes versus win outcomes, as hypothesized. Further, we obtained preliminary evidence that psychosocial intervention is associated with altered feedback processing. Specifically, FRN amplitudes were reduced (less negative) in minimal-intervention participants compared with extended-intervention participants, suggesting that the minimal-intervention group was physiologically hypo-sensitive to feedback. The fact that the FRN was significantly larger in the extended-intervention group suggests the possibility that feedback processing (at least as reflected by this measure) may be improved or normalized following intervention. As reviewed above, a number of studies indicate that externalizing adolescents are deficient in processing feedback information [for example, 67] and show reduced FRN amplitudes [30,31]. By contrast, the FRN did not differ between groups of violent versus non-violent adult men in a study by Wiswede et al. [50]. The current data therefore support and extend previous research with young people, by demonstrating that at-risk adolescents who have not yet received psychosocial intervention show reduced FRN amplitudes compared with those who have engaged in the intervention.

The finding that the FRN differed between the minimal-intervention and extended-intervention groups suggests that this ERP component may be a useful biomarker of psychosocial intervention. A number of existing studies have used the FRN (or variations of it) to assess numerous intervention styles, including mindfulness [68,69], and cognitive behavioural therapy [70]. The FRN has been used to track intervention-related change in populations including adults with attention deficit hyperactivity disorder [69], children and young adults with generalized or social anxiety disorders [70], adolescents with dyslexia [71] and healthy adults [68]. Our study therefore extends the utility of the FRN to at-risk adolescent populations, and to community-based psychosocial interventions.

In our study, neither FRN nor P3 amplitudes were sensitive to provocation. Previous research investigating whether FRN amplitudes are impacted by provocation is mixed. Krämer et al. [33] report that FRN amplitudes in adult participants are not modulated by provocation whereas we found reduced FRN amplitudes during high provocation in 
typically-developing adolescents [3]. Outside of the TAP task, research investigating the FRN's sensitivity to feedback magnitude is also inconsistent $[65,66,72,43]$. Further research is clearly needed to uncover the conditions under which the FRN and the P3 are sensitive to subtle variations in the nature of the feedback provided.

Following previous research, the P3 component was larger (more positive) for win outcomes compared with loss outcomes [39-43]. Of greater interest was the interaction between outcome (win versus loss) and group. The difference between win and loss outcomes was much smaller for the minimal-intervention group, compared with the extended-intervention group, suggesting hypo-sensitivity in the minimal-intervention participants. This hypo-sensitivity to reward can be linked to prior work, which argues that reward processing is deficient in young people with antisocial behavior [for example, 22,27,21]. In a novel contribution to the literature, our current data suggests that neural hyposensitivity in at-risk adolescents may be overcome using psychosocial intervention.

As well as physiological hypo-sensitivity to outcome stimuli, the current data revealed that P3 amplitudes were generally smaller in the minimal-intervention group. Reduced P3 amplitudes have been linked to psychopathologies including externalizing disorders, depression, schizophrenia and dependency disorders [34,36-38,73]. For example, reduced P3 amplitudes have been observed in impulsively violent adults [74] and maturation of the P3 is impaired in young males with conduct problems [35]. Longitudinal data indicate that small P3 amplitudes in childhood are predictive of substance abuse [38] and criminality in adulthood [36]. Interestingly, previous studies show that P3 amplitudes can be normalized through psychological intervention in various patient populations (including adults with depression, cognitive impairment and Tic disorders) [75-77]. The cross-sectional design of our study limits us from drawing such strong conclusions about the role of the P3. However, the finding that P3 amplitudes were larger in atrisk adolescents who had received extensive psychosocial intervention may suggest that P3 amplitudes (and the underlying processes that the P3 indexes) are not fixed but can be altered via therapeutic intervention in at-risk adolescents. Longitudinal work will be required to substantiate this possibility.

\section{Limitations}

Although the current study makes a useful contribution to the literature, a number of limitations must be acknowledged. The study was designed as a 'proof of principle' analysis of psychosocial intervention in at-risk adolescents. Therefore, we would stress that no claims of causality should be drawn from this work. Longitudinal research and randomized controlled trials are essential for causal arguments to be made regarding intervention-related change in decision making and feedback processing. The cross-sectional nature of the study means participants were not randomly allocated to treatment groups, so it is possible that pre-existing group differences - rather than the intervention per se - were responsible for the obtained pattern of results. Further longitudinal work and randomized interventions will be vital to validate the findings from the current study. A further point of note is that at-risk 
adolescents are not a homogenous group and are not definable by psychiatric diagnostic criteria. Instead, the sample reported here reflects a population with a broad range of needs. This aspect of the study is both a strength and a weakness. On the one hand, the problems facing at-risk youth may be contextually-specific, such that research with one group of at-risk adolescents may not translate to another environment. On the other hand, however, community studies into disadvantaged youth are uncommon [but see 5], and more research of this nature is needed to help understand and improve outcomes in this sector of society. This is particularly true given that adolescent mental health services often cater for clients with diverse social and psychological needs, who cannot be strictly classified using psychiatric diagnostic manuals. Finally, all participants were male and it is unclear whether the results would equally apply to female participants. Additional research that specifically includes adolescent girls will be helpful to understand whether the current findings are gender specific.

\section{Further Comments}

Our study investigated the in decision making and feedback processing associated with psychosocial intervention in at-risk adolescents. The findings also speak, pragmatically, to the way in which intervention-based research on social processing and decision making should be conducted with at-risk young people. Through the use of an engaging, realistic task, we were able to collect high-quality data from adolescents who are often difficult to engage in research. Future work should therefore continue to develop tasks that young people find enjoyable, but which also probe cognitive functions of interest. An additional pragmatic consideration to emerge from this study is that ERP measures appear to be sensitive enough to highlight intervention-related group differences. This study therefore contributes to the small literature showing that EEG can help characterize the mechanisms associated with therapeutic change in hard-to-reach populations $[16,18]$. We specifically employed a portable EEG system, which enabled data collection to occur in community centres that were familiar to participants, and potentially increased engagement in the study.

In summary, here we report the first brain-based investigation of decision making and feedback processing in adolescents exposed to multiple social disadvantages. Collectively, the data suggest that performance on a competitive social task may be improved as a result of engagement in psychosocial intervention, and that such a hypothesis warrants fully randomized controlled trials. Group differences were observed for a number of processes including emotional reactivity during the Decision Phase (as indexed by the LPP), feedback processing (as indexed by the FRN) and reward sensitivity (as indexed by the P3), suggesting that the psychosocial intervention may had numerous psychological and interpersonal benefits. These data have the potential to enhance the development and evaluation of psychological interventions in at-risk young people. 


\section{Ethical Standards}

Ethical approval for the study was provided by the Research Ethics Committee at University College London (ID: 3064/001), and debriefing occurred at the end of the study. This study has been performed in accordance with 1964 Declaration of Helsinki, and its later amendments.

\section{Conflict of interest}

This study was supported by the Waterloo Foundation and Kids Company. The Waterloo Foundation had no role in study design, data collection and analysis, the decision to publish, or preparation of the manuscript. Kids Company supported the recruitment of participants from their services, and provided lab space, equipment and top-up funding. Kids Company had no role in study design, data collection and analysis, the decision to publish, or preparation of the manuscript. Professor Peter Fonagy is chief executive of Anna Freud National Centre for Children and Families (a child mental health charity). 


\section{References}

1. Moffitt TE, Caspi A, Rutter M, Silva PA (2001) Sex differences in antisocial behaviour: Conduct disorder, delinquency, and violence in the Dunedin Longitudinal Study. Cambridge University Press, Cambridge

2. Scott S, Knapp M, Henderson J, Maughan B (2001) Financial cost of social exclusion: follow up study of antisocial children into adulthood. British Medical Journal 323 (7306):191-194

3. Pincham HL, Wu C, Killikelly C, Vuillier L, Fearon RMP (2015) Social provocation modulates decision making and feedback processing: Examining the trajectory of development in adolescent participants. Developmental Cognitive Neuroscience 15:58-66

4. Taylor SP (1967) Aggressive behavior and physiological arousal as a function of provocation and the tendency to inhibit aggression. Journal of Personality 35 (2):297-310

5. Cecil CAM, Viding E, Barker ED, Guiney J, McCrory EJ (2014) Double disadvantage: The influence of childhood maltreatment and community violence exposure on adolescent mental health. Journal of Child Psychology and Psychiatry

6. Pincham HL, Bryce D, Kokorikou D, Fonagy P, Fearon RMP (2016) Psychosocial Intervention Is Associated with Altered Emotion Processing: An Event-Related Potential Study in At-Risk Adolescents. PloS one 11 (1):e0147357

7. McDermott JM, Troller-Renfree S, Vanderwert R, Nelson CA, Zeanah CH, Fox NA (2013) Psychosocial deprivation, executive functions, and the emergence of socio-emotional behavior problems. Frontiers in Human Neuroscience 7:167

8. McDermott JM, Westerlund A, Zeanah CH, Nelson CA, Fox NA (2012) Early adversity and neural correlates of executive function: Implications for academic adjustment. Developmental Cognitive Neuroscience 2:S59-S66 9. Pincham HL, Bryce D, Fearon RMP (2015) The neural correlates of emotion processing in juvenile offenders. Developmental Science 18 (6):994-1005. doi:10.1111

10. Alexander JF, Robbins MS, Sexton TL (2000) Family-based interventions with older, at-risk youth: From promise to proof to practice. Journal of Primary Prevention 21 (2):185-205

11. Cho H, Hallfors DD, Sánchez V (2005) Evaluation of a high school peer group intervention for at-risk youth. Journal of Abnormal Child Psychology 33 (3):363-374

12. Dishion TJ, Andrews DW (1995) Preventing escalation in problem behaviors with high-risk young adolescents: Immediate and 1-year outcomes. Journal of Consulting and Clinical Psychology 63 (4):538

13. Eggert LL, Thompson EA, Herting JR, Nicholas LJ (1995) Reducing Suicide Potential Among High-Risk Youth: Tests of a School-Based Prevention Program. Suicide and Life-Threatening Behavior 25 (2):276-296

14. Keating LM, Tomishima MA, Foster S, Alessandri M (2002) The effects of a mentoring program on at-risk youth. Adolescence 37 (148):717-734

15. Moody KA, Childs JC, Sepples SB (2002) Intervening with at-risk youth: evaluation of the youth empowerment and support program. Pediatric Nursing 29 (4):263-270

16. Lewis MD, Granic I, Lamm C, Zelazo PD, Stieben J, Todd RM, Moadab I, Pepler D (2008) Changes in the neural bases of emotion regulation associated with clinical improvement in children with behavior problems. Development and Psychopathology 20 (03):913-939

17. Roffman JL, Marci CD, Glick DM, Dougherty DD, Rauch SL (2005) Neuroimaging and the functional neuroanatomy of psychotherapy. Psychological Medicine 35 (10):1385-1398

18. Woltering S, Granic I, Lamm C, Lewis MD (2011) Neural changes associated with treatment outcome in children with externalizing problems. Biological Psychiatry 70 (9):873-879

19. Shaw DS, Shelleby EC (2014) Early-Starting Conduct Problems: Intersection of Conduct Problems and Poverty. Annual Review of Clinical Psychology 10

20. Shonkoff JP, Garner AS (2012) Committee on Psychosocial Aspects of Child and Family Health; Committee on Early Childhood, Adoption, and Dependent Care; Section on Developmental and Behavioral Pediatrics. The lifelong effects of early childhood adversity and toxic stress. Pediatrics 129 (1):e232-e246

21. Lane SD, Cherek DR (2001) Risk taking by adolescents with maladaptive behavior histories. Experimental and Clinical Psychopharmacology 9 (1):74

22. Fonseca A, Yule W (1995) Personality and antisocial behavior in children and adolescents: an enquiry into Eysenck's and Gray's theories. Journal of Abnormal Child Psychology 23 (6):767-781

23. Matthys W, van Goozen SH, Vries Hd, Cohen-Kettenis PT, Engeland Hv (1998) The dominance of behavioural activation over behavioural inhibition in conduct disordered boys with or without attention deficit hyperactivity disorder. Journal of Child Psychology and Psychiatry 39 (5):643-651

24. Bjork JM, Chen G, Smith AR, Hommer DW (2010) Incentive-elicited mesolimbic activation and externalizing symptomatology in adolescents. Journal of Child Psychology and Psychiatry 51 (7):827-837

25. Finger EC, Marsh AA, Mitchell DG, Reid ME, Sims C, Budhani S, Kosson DS, Chen G, Towbin KE, Leibenluft E (2008) Abnormal ventromedial prefrontal cortex function in children with psychopathic traits during reversal learning. Archives of General Psychiatry 65 (5):586-594

26. White SF, Brislin S, Sinclair S, Fowler KA, Pope K, Blair RJR (2013) The relationship between large cavum septum pellucidum and antisocial behavior, callous-unemotional traits and psychopathy in adolescents. Journal of Child Psychology and Psychiatry 54 (5):575-581 
27. Gatzke-Kopp LM, Beauchaine TP, Shannon KE, Chipman J, Fleming AP, Crowell SE, Liang O, Johnson LC, Aylward E (2009) Neurological correlates of reward responding in adolescents with and without externalizing behavior disorders. Journal of Abnormal Psychology 118 (1):203-213

28. Finger EC, Marsh AA, Blair KS, Reid ME, Sims C, Ng P, Pine DS, Blair RJR (2011) Disrupted reinforcement signaling in the orbitofrontal cortex and caudate in youths with conduct disorder or oppositional defiant disorder and a high level of psychopathic traits. American Journal of Psychiatry 168 (2):152-162

29. Rubia K, Smith A, Halari R, Matsukura F, Mohammad M, Taylor E, Brammer M (2009) Disorder-specific dissociation of orbitofrontal dysfunction in boys with pure conduct disorder during reward and ventrolateral prefrontal dysfunction in boys with pure ADHD during sustained attention. American Journal of Psychiatry 166 (1):83-94 30. Crowley MJ, Wu J, Crutcher C, Bailey CA, Lejuez C, Mayes LC (2009) Risk-taking and the feedback negativity response to loss among at-risk adolescents. Developmental Neuroscience 31 (1-2):137-148

31. Segalowitz SJ, Santesso DL, Willoughby T, Reker DL, Campbell K, Chalmers H, Rose-Krasnor L (2012) Adolescent peer interaction and trait surgency weaken medial prefrontal cortex responses to failure. Social Cognitive and Affective Neuroscience 7 (1):115-124

32. Van Leijenhorst L, Zanolie K, Van Meel CS, Westenberg PM, Rombouts SA, Crone EA (2009) What motivates the adolescent? Brain regions mediating reward sensitivity across adolescence. Cerebral cortex 20 (1):61-69

33. Krämer UM, Büttner S, Roth G, Münte TF (2008) Trait aggressiveness modulates neurophysiological correlates of laboratory-induced reactive aggression in humans. Journal of Cognitive Neuroscience 20 (8):1464-1477

34. Bauer LO, Hesselbrock VM (1999) P300 decrements in teenagers with conduct problems: implications for substance abuse risk and brain development. Biological Psychiatry 46 (2):263-272

35. Bauer LO, Hesselbrock VM (2003) Brain maturation and subtypes of conduct disorder: interactive effects on P300 amplitude and topography in male adolescents. Journal of the American Academy of Child \& Adolescent Psychiatry 42 (1):106-115

36. Gao Y, Raine A, Venables PH, Mednick SA (2013) The Association Between P3 Amplitude at Age 11 and Criminal Offending at Age 23. Journal of Clinical Child \& Adolescent Psychology 42 (1):120-130

37. Hicks BM, Bernat E, Malone SM, Iacono WG, Patrick CJ, Krueger RF, McGue M (2007) Genes mediate the association between P3 amplitude and externalizing disorders. Psychophysiology 44 (1):98-105

38. Iacono WG, Carlson SR, Malone SM, McGue M (2002) P3 event-related potential amplitude and the risk for disinhibitory disorders in adolescent boys. Archives of General Psychiatry 59 (8):750-757

39. Bellebaum C, Polezzi D, Daum I (2010) It is less than you expected: the feedback-related negativity reflects violations of reward magnitude expectations. Neuropsychologia 48 (11):3343-3350

40. Hajcak G, Holroyd CB, Moser JS, Simons RF (2005) Brain potentials associated with expected and unexpected good and bad outcomes. Psychophysiology 42 (2):161-170

41. Hajcak G, Moser JS, Holroyd CB, Simons RF (2007) It's worse than you thought: The feedback negativity and violations of reward prediction in gambling tasks. Psychophysiology 44 (6):905-912

42. Holroyd CB, Hajcak G, Larsen JT (2006) The good, the bad and the neutral: electrophysiological responses to feedback stimuli. Brain Research 1105 (1):93-101

43. Wu Y, Zhou X (2009) The P300 and reward valence, magnitude, and expectancy in outcome evaluation. Brain Research 1286:114-122

44. Gaskell C (2008) Kids Company help with the whole problem. Kids Company Research and Evaluation Programme

45. Jovchelovitch S, Concha N (2013) Kids Company: a diagnosis of the organisation and its interventions: executive summary.

46. Capaldi DM, Rothbart MK (1992) Development and validation of an early adolescent temperament measure. The Journal of Early Adolescence 12 (2):153-173

47. Goodman R (1997) The Strengths and Difficulties Questionnaire: a research note. Journal of child psychology and psychiatry 38 (5):581-586

48. Essau CA, Sasagawa S, Frick PJ (2006) Callous-unemotional traits in a community sample of adolescents. Assessment 13 (4):454-469

49. Nolan H, Whelan R, Reilly R (2010) FASTER: Fully Automated Statistical Thresholding for EEG artifact Rejection. Journal of Neuroscience Methods 192 (1):152-162

50. Wiswede D, Taubner S, Münte TF, Roth G, Strüber D, Wahl K, Krämer UM (2011) Neurophysiological correlates of laboratory-induced aggression in young men with and without a history of violence. PloS One 6 (7):e22599 51. Cuthbert BN, Schupp HT, Bradley MM, Birbaumer N, Lang PJ (2000) Brain potentials in affective picture processing: covariation with autonomic arousal and affective report. Biological Psychology 52 (2):95-111 52. Yeung N, Sanfey AG (2004) Independent coding of reward magnitude and valence in the human brain. The Journal of Neuroscience 24 (28):6258-6264

53. Zottoli TM, Grose-Fifer J (2012) The feedback-related negativity (FRN) in adolescents. Psychophysiology 49 (3):413-420

54. Massar S, Rossi V, Schutter D, Kenemans J (2012) Baseline EEG theta/beta ratio and punishment sensitivity as biomarkers for feedback-related negativity (FRN) and risk-taking. Clinical Neurophysiology 123 (10):1958-1965 55. Potts GF, Martin LE, Burton P, Montague PR (2006) When things are better or worse than expected: the medial frontal cortex and the allocation of processing resources. Journal of Cognitive Neuroscience 18 (7):1112-1119 
56. Smillie LD, Cooper AJ, Pickering AD (2011) Individual differences in reward-prediction-error: extraversion and feedback-related negativity. Social Cognitive and Affective Neuroscience 6 (5):646-652

57. Cui J-f, Chen Y-h, Wang Y, Shum DH, Chan RC (2013) Neural correlates of uncertain decision making: ERP evidence from the Iowa Gambling Task. Frontiers in Human Neuroscience 7

58. Gu R, Wu T, Jiang Y, Luo YJ (2011) Woulda, coulda, shoulda: the evaluation and the impact of the alternative outcome. Psychophysiology 48 (10):1354-1360

59. Martin LE, Potts GF (2009) Impulsivity in decision-making: An event-related potential investigation. Personality and Individual Differences 46 (3):303-308

60. Connor DF, Carlson GA, Chang KD, Daniolos PT, Ferziger R, Findling RL, Hutchinson JG, Malone RP, Halperin JM, Plattner B (2006) Juvenile maladaptive aggression: a review of prevention, treatment, and service configuration and a proposed research agenda. The Journal of Clinical Psychiatry 67 (5):808-820

61. Bradley MM, Hamby S, Löw A, Lang PJ (2007) Brain potentials in perception: picture complexity and emotional arousal. Psychophysiology 44 (3):364-373

62. Codispoti M, Ferrari V, Bradley MM (2007) Repetition and event-related potentials: Distinguishing early and late processes in affective picture perception. Journal of Cognitive Neuroscience 19 (4):577-586

63. Naumann E, D. Bartussek, Diedrich O., Laufer M.E. (1992) Assessing cognitive and affective information processing functions of the brain by means of the late positive complex of the event-related potential. Journal of Psychophysiology 6 (4):285-298

64. Gehring WJ, Willoughby AR (2002) The medial frontal cortex and the rapid processing of monetary gains and losses. Science 295 (5563):2279-2282. doi:10.1126/science.1066893

295/5563/2279 [pii]

65. Goyer JP, Woldorff MG, Huettel SA (2008) Rapid electrophysiological brain responses are influenced by both valence and magnitude of monetary rewards. Journal of Cognitive Neuroscience 20 (11):2058-2069

66. Hajcak G, Moser JS, Holroyd CB, Simons RF (2006) The feedback-related negativity reflects the binary

evaluation of good versus bad outcomes. Biological Psychology 71 (2):148-154. doi:S0301-0511(05)00079-7 [pii]

10.1016/j.biopsycho.2005.04.001

67. Steinberg L (2008) A social neuroscience perspective on adolescent risk-taking. Developmental Review 28 (1):78106

68. Larson MJ, Steffen PR, Primosch M (2013) The impact of a brief mindfulness meditation intervention on cognitive control and error-related performance monitoring. Frontiers in Human Neuroscience 7

69. Schoenberg PL, Hepark S, Kan CC, Barendregt HP, Buitelaar JK, Speckens AE (2014) Effects of mindfulnessbased cognitive therapy on neurophysiological correlates of performance monitoring in adult attention-

deficit/hyperactivity disorder. Clinical Neurophysiology 125 (7):1407-1416

70. Kujawa A, Weinberg A, Bunford N, Fitzgerald KD, Hanna GL, Monk CS, Kennedy AE, Klumpp H, Hajcak G, Phan KL (2016) Error-related brain activity in youth and young adults before and after treatment for generalized or social anxiety disorder. Progress in Neuro-Psychopharmacology and Biological Psychiatry 71:162-168

71. Horowitz-Kraus T (2016) Improvement of the Error-detection Mechanism in Adults with Dyslexia Following Reading Acceleration Training. Dyslexia 22 (2):173-189

72. Toyomaki A, Murohashi H (2005) Discrepancy between feedback negativity and subjective evaluation in gambling. NeuroReport 16 (16):1865-1868

73. Schupp HT, Markus J, Weike AI, Hamm AO (2003) Emotional facilitation of sensory processing in the visual cortex. Psychological Science 14 (1):7-13

74. Zukov I, Ptacek R, Kozelek P, Fischer S, Domluvilova D, Raboch J, Hruby T, Susta M (2009) Brain wave P300: A comparative study of various forms of criminal activity. Medical Science Monitor 15 (7):CR349-354

75. Morand-Beaulieu S, O'Connor KP, Sauvé G, Blanchet PJ, Lavoie ME (2015) Cognitive-behavioral therapy induces sensorimotor and specific electrocortical changes in chronic tic and Tourette's disorder. Neuropsychologia 79:310-321

76. Naga Venkatesha Murthy P, Gangadhar B, Janakiramaiah N, Subbakrishna D (1997) Normalization of P300 amplitude following treatment in dysthymia. Biological Psychiatry 42 (8):740-743

77. Smart CM (2014) Mindfulness training: A novel approach to intervening in older adults with subjective cognitive decline. Alzheimer's \& Dementia: The Journal of the Alzheimer's Association 10 (4):P164 